\title{
Phase Prediction of the Response of Choked Nozzles to Entropy and Acoustic Disturbances
}

\author{
Chee Su Goh*, Aimee S. Morgans \\ Department of Aeronautics, Imperial College London, UK
}

\begin{abstract}
The development and transmission of sound through the exit of an aeroengine combustor is often investigated by modelling the complex geometry as a convergent-divergent nozzle. However, these analytical acoustic predictions are usually limited to the compact case, where the length of the nozzle is insignificant compared to the wavelength of the flow perturbations, or to cases where the variation of the mean velocity through the nozzle may be treated as linear, or piece-wise linear. Considering terms up to first order in frequency for the conservation of mass, momentum and energy, this paper investigates an alternative approach by deriving effective lengths for the passage of the flow perturbations through a supercritical convergent-divergent nozzle. The effects due to the presence of a normal shock wave are also studied using a linearised form of the Rankine-Hugoniot relations. The analyses lead to predictions for the phase and magnitude of the transmitted acoustic waves from finite-length nozzles, and are valid for low non-dimensional frequencies. It has been found that these predictions agree well with the numerical results from inviscid simulations.
\end{abstract}

\section{Introduction}

A significant source of noise created by propulsive devices, such as jet engines and ramjets, is due to unsteady combustion. The fluctuating heat release within the combustor generates acoustic perturbations (known as direct combustion noise) as a monopole source [1], as well as temperature 'hot spots' or entropy waves. Unlike the direct combustion noise, entropy waves are quiescent in non-accelerating flow, and convect with the mean flow velocity. However, a fluctuating force is required to accelerate these entropy waves, such as through the turbine stages of a jet engine, which constitutes a dipole source of sound $[2,3]$. The acoustics caused by this secondary mechanism is known as entropy noise, or indirect combustion noise. Combustion noise has been found to be

\footnotetext{
${ }^{*}$ Corresponding author

Email address: cheesu.goh08@imperial.ac.uk (Chee Su Goh)
} 
important at low frequencies [4], with entropy noise dominating over direct combustion noise for up to $1 \mathrm{kHz}$ as described by Cumpsty [5].

The phenomenon of sound generation due to flow inhomogeneities was qualitatively described by Lord Rayleigh in chapter XV of his 1896 book [6]. However, it was not until almost 80 years later that Morfey established a mathematical framework explaining how the acceleration of non-uniform density perturbations scattered sound [7]. These entropy inhomogeneities were incorporated into Lighthill's acoustic analogy by Howe [2], who, together with Ffowcs Williams, predicted the acoustic 'bremsstrahlung' due to sharp-fronted inhomogeneities in low Mach number flows using Green's functions $[8,9]$.

In order to avoid the complications of the Green's function technique, alternative one-dimensional analyses were performed for the compact nozzle, where all perturbation wavelengths are much longer than the length of the nozzle; implying that the perturbations are effectively accelerated through an abrupt area change. Acoustic response coefficients for the subsonic $[10,11]$ and supercritical [11] nozzles were derived and found to be a function of Mach numbers alone. The compact coefficients provided a good analytical prediction at very low frequencies. Extending the work by Marble and Candel [11], Stow et al. [12] and Moase et al. [13] incorporated the effect of a normal shock wave oscillating with a small amplitude within the diffuser of a convergent-divergent nozzle. They utilised the linearised Rankine-Hugoniot methodology previously adopted by Culick and Rogers [14], and Kuo and Dowling [15].

These compact transmission and reflection coefficients may be used to predict the acoustic response of several successive stages of turbine blades. Cumpsty and Marble [16] proposed a matrix expression for the behaviour between blade rows in order to predict low-frequency engine core noise. They found that the results agreed well with acoustic data collated from the Rolls-Royce Spey 512, Olympus 593 and Pratt and Whitney JT8D-9 gas turbines [17]. Another application for these compact coefficients is in the frequency-domain low order 'network models' used to study the linear stability of complex thermoacoustic systems, as described in Sattelmayer and Polifke [18]. The compact results have even been employed in astrophysics to study a possible mechanism for the instability of shocked accretion flows onto a star [19].

In spite of further numerical $[20,21]$ and experimental $[17,22]$ support for the theoretical analyses, the importance of indirect combustion noise compared to the direct mechanism in gas turbines has been highly debated. In an experiment with a general combustion chamber, Eckstein et al. [23] concluded that the acoustic response of entropy waves was insignificant due to their highly dispersive nature. Sattelmayer [24] showed that the dispersion of the entropy waves could be taken into account via an analytical approach. Furthermore, the noise data from gas turbine auxiliary power units led Tam et al. [25] to claim that there was no evidence for the indirect mechanism. Dowling and Hubbard [26] admitted that entropy waves could have possibly diffused prior to reaching the combustor exit, and hence, the generation of entropy noise would be more significant in aero-engines and aeroderivative gas turbines with shorter combustors compared to industrial-type turbines. 
Part of the uncertainty may be due to the difficulty to distinguish between direct and indirect combustion noise experimentally. In an attempt to separate the two, Muthukrishnan et al. [27] performed a statistical coherence analysis on near and far-field signals from a combustor, and found that entropy noise was dominant when there was a high Mach number gradient at the exit. Recent canonical experiments by Bake et al. using electrical heating impulses provided strong evidence for entropy noise [28, 29, 30]. Their conclusions were further corroborated by the predictions from Howe's analytical approach using the acoustic analogy theory [3]. The analysis suggested that the transmitted sound level was reduced by the interaction of entropy noise with the vorticity from the flow-separated jet in the diffuser, and by the reduction of the entropy gradient due to the streamwise stretching of the entropy disturbance as it accelerates through the nozzle. (In the present study, the vorticity mechanism has been ignored as it is assumed that the boundary layer in the diffuser remains attached, in line with the flow behaviour through the nozzle guide vanes at the exit of a gas turbine combustor. Whilst being an important factor, this work does not include the effects of entropy wave stretching.)

To extend the theoretical analysis beyond the compact behaviour, Marble and Candel investigated frequency effects in a finite-length nozzle in the second half of their seminal paper [11]. Nevertheless, the analysis was limited to supercritical nozzles where the mean velocity distribution could be assumed to be linear with no shock present. Moase et al. [13] extended this approach to the case where the steady velocity is piece-wise linear, deriving the matching conditions required between the linear segments of the nozzle, which also allowed for the presence of a shock. An alternative to the linear velocity assumption to study frequency effects was suggested by Stow et al. [12]. They used an analytical technique, valid for low frequencies, to determine a phase correction applied to the reflection coefficients of a compact choked nozzle.

The aim of this paper is to extend the analysis performed by Stow et al. [12] to the transmitted acoustic response of a nozzle. The next section outlines the asymptotic analysis of flow perturbations using the equations for the conservation of mass, momentum and energy. In Section 3, the transmission effective length for a supercritical convergent-divergent nozzle is derived. The expression for the effective length is found to be dependent on the input perturbation type, i.e. either acoustic or entropic, and is used to improve the compact transmission prediction with a phase correction accurate to first-order in frequency. The analytical results are then compared with numerical data from an Euler code. In Section 4, a linearised version of the Rankine-Hugoniot relations is adapted to predict the behaviour of the response downstream of a shock. It is found that there is good agreement between the analytical phase predictions and the numerical results. 


\section{Analysis}

\subsection{Governing flow equations}

Away from any heat source, the conservation of mass, momentum and energy applied to a perfect gas may be written as

$$
\begin{aligned}
\frac{\partial \rho}{\partial t}+\nabla \cdot(\rho \mathbf{u}) & =0, \\
\rho \frac{\mathrm{D} \mathbf{u}}{\mathrm{D} t}+\nabla p & =\mathbf{0}, \\
\frac{\mathrm{D} s}{\mathrm{D} t} & =0,
\end{aligned}
$$

where the flow phenomena being studied is assumed to be inviscid. Here $\rho$ is density, $\mathbf{u}$ the velocity vector, $p$ pressure, $s$ entropy, and $t$ time.

For a flow through a duct of constant cross-sectional area, Dowling and Stow [31] showed that by re-writing the flow properties as a sum of time-averaged (denoted with an overbar) and fluctuating (denoted with a prime) components, the linearised conservation equations may be rearranged to describe the development of acoustic, entropy and vorticity fluctuations. These are summarised below:

1. Isentropic and irrotational acoustic fluctuations,

$$
\frac{1}{\bar{c}^{2}} \frac{\overline{\mathrm{D}}^{2} p^{\prime}}{\mathrm{D} t^{2}}-\nabla^{2} p^{\prime}=0 .
$$

2. Incompressible and irrotational entropy fluctuations,

$$
\frac{\overline{\mathrm{D}} s^{\prime}}{\mathrm{D} t}=0 .
$$

3. Incompressible and isentropic vorticity fluctuations,

$$
\frac{\overline{\mathrm{D}} \xi^{\prime}}{\mathrm{D} t}=\mathbf{0},
$$

where $\xi$ is the vorticity.

The linearised governing equations for the three types of perturbations indicate that they are uncoupled in non-accelerating flow, and that the acoustic waves propagate with the speed of sound relative to the mean flow, whilst entropy and vorticity fluctuations advect with the mean flow speed. In this study, we are mainly concerned with acoustic and entropy fluctuations.

The acoustic waves may be expressed as planar waves propagating both downstream and upstream relative to the steady flow. In the one-dimensional case, with $x$ representing the spatial domain, and $\omega$ the angular frequency,

$$
\begin{aligned}
\frac{p^{\prime}}{\gamma \bar{p}} & =P^{+} \exp \left(\mathrm{i} \omega\left[t-\frac{x}{\bar{c}+\bar{u}}\right]\right)+P^{-} \exp \left(\mathrm{i} \omega\left[t+\frac{x}{\bar{c}-\bar{u}}\right]\right), \\
\frac{u^{\prime}}{\bar{u}} & =\frac{1}{\bar{M}} P^{+} \exp \left(\mathrm{i} \omega\left[t-\frac{x}{\bar{c}+\bar{u}}\right]\right)-\frac{1}{\bar{M}} P^{-} \exp \left(\mathrm{i} \omega\left[t+\frac{x}{\bar{c}-\bar{u}}\right]\right),
\end{aligned}
$$


where the superscript + indicates downstream-travelling and - upstream-travelling waves relative to the mean flow speed.

When gradients in steady flow properties exists, such as the passage through a nozzle, the perturbations described above are no longer uncoupled. Entropy waves are converted into acoustic energy as they accelerate through the nozzle. This results in both reflected and transmitted acoustic waves. The linearised mass, momentum and energy conservation equations (1a) to (1c) in one spatial dimension may be written as

$$
\begin{aligned}
\frac{\partial \rho^{\prime}}{\partial t}+\bar{\rho} \frac{\partial u^{\prime}}{\partial x}+\rho^{\prime} \frac{\partial \bar{u}}{\partial x}+\bar{u} \frac{\partial \rho^{\prime}}{\partial x}+u^{\prime} \frac{\partial \bar{\rho}}{\partial x} & =0 \\
\bar{\rho} \frac{\partial u^{\prime}}{\partial t}+\bar{\rho} \bar{u} \frac{\partial u^{\prime}}{\partial x}+\left(\bar{\rho} u^{\prime}+\bar{u} \rho^{\prime}\right) \frac{\partial \bar{u}}{\partial x}+\frac{\partial p^{\prime}}{\partial x} & =0, \\
\frac{\partial s^{\prime}}{\partial t}+\bar{u} \frac{\partial s^{\prime}}{\partial x} & =0 .
\end{aligned}
$$

The flow perturbations may be assumed to have an $e^{\mathrm{i} \omega t}$ temporal dependence, and written in non-dimensional form:

$$
\frac{p^{\prime}}{\gamma \bar{p}}=\hat{p}(X) \mathrm{e}^{\mathrm{i} \omega t}, \quad \frac{\rho^{\prime}}{\bar{\rho}}=\hat{\rho}(X) \mathrm{e}^{\mathrm{i} \omega t}, \quad \frac{u^{\prime}}{\bar{u}}=\hat{u}(X) \mathrm{e}^{\mathrm{i} \omega t},
$$

where $X=x / L$, and $L$ is a characteristic axial length of the nozzle.

\subsection{Asymptotic analysis for a choked nozzle}

If the nozzle is choked, the Mach number and perturbation frequency may be non-dimensionalised with reference to the speed of sound $c$ at the nozzle throat;

$$
M^{*}=\frac{\bar{u}}{c^{*}}, \quad \Omega=\frac{\omega L}{c^{*}},
$$

where the superscript $*$ denotes the choked nozzle throat location, $M$ the Mach number, and $\Omega$ the non-dimensional frequency which also serves as a measure of the nozzle compactness.

After removing the steady flow components, the mass continuity equation (3a) may be recast as

$$
\mathrm{i} \Omega \hat{\rho}+M^{*}\left(\frac{\mathrm{d} \hat{u}}{\mathrm{~d} X}+\frac{\mathrm{d} \hat{\rho}}{\mathrm{d} X}\right)=0 .
$$

Given that the conservation of total enthalpy dictates that

$$
\bar{c}^{2}=\frac{1}{2}(\gamma+1) c^{* 2}-\frac{1}{2}(\gamma-1) \bar{u}^{2},
$$

the momentum equation $(3 \mathrm{~b})$ can be re-written as

$$
\mathrm{i} \Omega \hat{u}+M^{*} \frac{\mathrm{d} \hat{u}}{\mathrm{~d} X}+\frac{\mathrm{d} M^{*}}{\mathrm{~d} X}(2 \hat{u}+\hat{\rho}-\gamma \hat{p})+\frac{\mathrm{d} \hat{p}}{\mathrm{~d} X} \frac{1}{2}\left(\frac{(\gamma+1)}{M^{*}}-(\gamma-1) M^{*}\right)=0 .
$$


Similarly, using the relation $\sigma=s^{\prime} / c_{p}=p^{\prime} /(\gamma \bar{p})-\rho^{\prime} / \bar{\rho},\left(c_{p}\right.$ being the specific heat capacity at constant pressure), the energy equation (3c) can be expressed in non-dimensional form:

$$
\mathrm{i} \Omega(\hat{p}-\hat{\rho})+M^{*}\left(\frac{\mathrm{d} \hat{p}}{\mathrm{~d} X}-\frac{\mathrm{d} \hat{\rho}}{\mathrm{d} X}\right)=0 .
$$

In order to study the development of the flow perturbations, the conservation equations may be combined by multiplying equation (5) by $2 M^{*}$, and subtracting $2 / M^{*}$ times $(4)$ and $\left(M^{*}+1 / M^{*}\right)$ times (6), giving

$$
\mathrm{i} \Omega\left[M^{*}(2 \hat{u}+\hat{\rho}-\hat{p})-\frac{1}{M^{*}}(\hat{p}+\hat{\rho})\right]+\frac{\mathrm{d}}{\mathrm{d} X}\left[\left(M^{* 2}-1\right)(2 \hat{u}+\hat{\rho}-\gamma \hat{p})\right]=0 .
$$

To proceed further, the flow perturbations may be treated as asymptotic expansions for small $\Omega$,

$$
\begin{aligned}
& \hat{p}=\hat{p}_{0}+\mathrm{i} \Omega \hat{p}_{1}+O\left(\Omega^{2}\right), \\
& \hat{\rho}=\hat{\rho}_{0}+\mathrm{i} \Omega \hat{\rho}_{1}+O\left(\Omega^{2}\right), \\
& \hat{u}=\hat{u}_{0}+\mathrm{i} \Omega \hat{u}_{1}+O\left(\Omega^{2}\right) .
\end{aligned}
$$

Stow et al. [12] showed that after substituting these expansions into equation (7), the boundary condition due to the choked nozzle is obtained by comparing terms to zeroth order in $\Omega$ :

$$
2 \hat{u}_{0}+\hat{\rho}_{0}-\gamma \hat{p}_{0}=0 .
$$

To first order in $\Omega$, equation (7) may be integrated to give

$$
\begin{aligned}
{\left[( 1 - M ^ { * 2 } ) \left(2 \hat{u}_{1}(X)+\right.\right.} & \left.\left.\hat{\rho}_{1}(X)-\gamma \hat{p}_{1}(X)\right)\right]_{X_{1}}^{X_{2}} \\
& =\hat{p}_{0}(\gamma-1) \int_{X_{1}}^{X_{2}} M^{*} \mathrm{~d} X-\left(\hat{p}_{0}+\hat{\rho}_{0}\right) \int_{X_{1}}^{X_{2}} \frac{1}{M^{*}} \mathrm{~d} X,
\end{aligned}
$$

which will be applied in Section 3 to predict the phase response of a supercritical nozzle.

\section{The supercritical nozzle}

\subsection{The effective lengths for a supercritical nozzle}

With the aim of deriving an $O(\Omega)$ phase correction, equation (9) may be used to compare the convergent-divergent nozzle with one where the nozzle is approximated by straight ducts joined at the throat location, as illustrated in Figure 1. Here the flow exiting the nozzle is supersonic and there is no shock present. As they were primarily interested in the reflected response of the nozzle, Stow et al. [12] applied equation (9) between the nozzle inlet $X=X_{\text {in }}$ and the choked throat $X=X^{*}$. Due to the flow being subsonic in this region, it was 
(a)

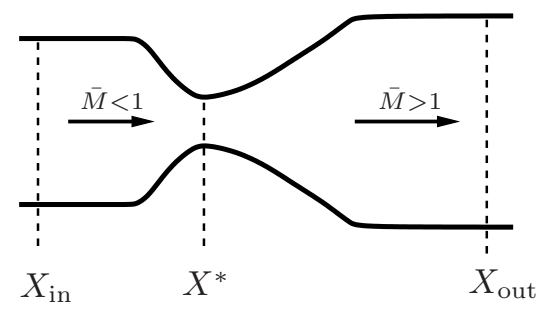

(b)

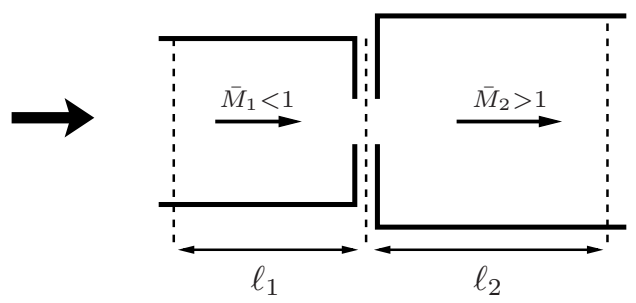

Figure 1: The (a) convergent-divergent nozzle is modelled by (b) ducts of constant areas, to obtain first order effective lengths, $\ell_{1}$ and $\ell_{2}$, for the flow perturbations.

shown that terms associated with $M^{*}$ could be neglected compared to terms with $1 / M^{*}$, and an effective length for the convergent section of the nozzle was obtained:

$$
\ell_{1}=\int_{X_{\mathrm{in}}}^{X^{*}} \frac{\bar{M}_{1}\left(1+\frac{1}{2}(\gamma-1) \bar{M}^{2}\right)^{\frac{1}{2}}}{\bar{M}\left(1+\frac{1}{2}(\gamma-1) \bar{M}_{1}^{2}\right)^{\frac{1}{2}}} \mathrm{~d} X
$$

where $\bar{M}_{1}$ is the inlet mean Mach number.

For the divergent section of the nozzle, equation (9) may be applied to the nozzle section between $X=X^{*}$ and $X=X_{\text {out }}$ (refer to Figure 1a),

$$
\begin{aligned}
2 \hat{u}\left(X_{\text {out }}\right)+\hat{\rho}\left(X_{\text {out }}\right)-\gamma \hat{p}\left(X_{\text {out }}\right)= \\
\quad \frac{\mathrm{i} \Omega}{M_{2}^{* 2}-1}\left[\left(\hat{p}_{0}+\hat{\rho}_{0}\right) \int_{X^{*}}^{X_{\text {out }}} \frac{1}{M^{*}} \mathrm{~d} X-\hat{p}_{0}(\gamma-1) \int_{X^{*}}^{X_{\text {out }}} M^{*} \mathrm{~d} X\right]
\end{aligned}
$$

where $M_{2}^{*}$ is a pseudo-Mach number at the nozzle exit. Similarly, an equivalent flow may be set up for the nozzle sketched in Figure 1b. Applying equation (9) between the nozzle throat and the exit, and denoting the equivalent flow with the accent ${ }^{\sim}$, gives

$$
\begin{aligned}
\left(1-M_{2}^{* 2}\right)\left[\left(2 \tilde{\hat{u}}_{1}\left(\tilde{X}_{\text {out }}\right)+\tilde{\hat{\rho}}_{1}\left(\tilde{X}_{\text {out }}\right)-\gamma \tilde{\hat{p}}_{1}\left(\tilde{X}_{\text {out }}\right)\right)\right. & \\
& \left.-\left(2 \tilde{\hat{u}}_{1}\left(X^{*}\right)+\tilde{\hat{\rho}}_{1}\left(X^{*}\right)-\gamma \tilde{\hat{p}}_{1}\left(X^{*}\right)\right)\right] \\
= & \hat{p}_{0}(\gamma-1) M_{2}^{*}\left(\tilde{X}_{\text {out }}-X^{*}\right)-\left(\hat{p}_{0}+\hat{\rho}_{0}\right) \frac{\tilde{X}_{\text {out }}-X^{*}}{M_{2}^{*}} .
\end{aligned}
$$

Equation (11) may be combined with (12), and noting that the effective length $\ell_{2}=\tilde{X}_{\text {out }}-X^{*}$,

$$
\ell_{2}=\frac{\left(\hat{p}_{0}+\hat{\rho}_{0}\right) \int_{X^{*}}^{X_{\mathrm{out}}} \frac{1}{M^{*}} \mathrm{~d} X-\hat{p}_{0}(\gamma-1) \int_{X^{*}}^{X_{\mathrm{out}}} M^{*} \mathrm{~d} X}{\frac{\hat{p}_{0}+\hat{\rho}_{0}}{M_{2}^{*}}-\hat{p}_{0}(\gamma-1) M_{2}^{*}} .
$$


In the case for $\ell_{1}$, the terms associated with $M^{*}$ in both the numerator and denominator were ignored, allowing $\hat{p}_{0}+\hat{\rho}_{0}$ to cancel out. However, this cannot be assumed for the divergent section of the nozzle where the flow is supersonic. Instead, we recall that $\hat{\rho}_{0}=\hat{p}_{0}-\sigma$, and use the Marble and Candel [11] expressions for the response of a compact supercritical nozzle to replace $\hat{p}_{0}$ with functions of Mach numbers alone. These compact relations depend on the type of input disturbance, either entropic or acoustic. For the entropy input case, the effective length for the divergent section is found to be

$$
\ell_{2, \sigma}=\frac{A_{1} \int_{X^{*}}^{X_{\text {out }}} \frac{\left(1+\frac{1}{2}(\gamma-1) \bar{M}^{2}\right)^{\frac{1}{2}}}{M} \mathrm{~d} X+A_{2} \int_{X^{*}}^{X_{\text {out }}} \frac{\bar{M}}{\left(1+\frac{1}{2}(\gamma-1) \bar{M}^{2}\right)^{\frac{1}{2}}} \mathrm{~d} X}{-\left(1+\frac{1}{2}(\gamma+1) \bar{M}_{1}\right)+\frac{1}{4} \frac{\bar{M}_{1} \bar{M}_{2}^{2}\left(\gamma^{2}-1\right)}{\left(1+\frac{1}{2}(\gamma-1) \bar{M}_{2}^{2}\right)}},
$$

where

$$
A_{1}=-\frac{\left(1+\frac{1}{2}(\gamma+1) \bar{M}_{1}\right) \bar{M}_{2}}{\left(1+\frac{1}{2}(\gamma-1) \bar{M}_{2}^{2}\right)^{\frac{1}{2}}}, \quad A_{2}=\frac{1}{4} \frac{\bar{M}_{1} \bar{M}_{2}\left(\gamma^{2}-1\right)}{\left(1+\frac{1}{2}(\gamma-1) \bar{M}_{2}^{2}\right)^{\frac{1}{2}}} .
$$

Therefore the expression for $\ell_{2}$, unlike that for $\ell_{1}$, is dependent on the type of input disturbance. When this is an acoustic perturbation,

$$
\ell_{2, p}=\frac{A_{3} \int_{X^{*}}^{X_{\text {out }}} \frac{\left(1+\frac{1}{2}(\gamma-1) \bar{M}^{2}\right)^{\frac{1}{2}}}{M} \mathrm{~d} X+A_{4} \int_{X^{*}}^{X_{\text {out }}} \frac{\bar{M}}{\left(1+\frac{1}{2}(\gamma-1) \bar{M}^{2}\right)^{\frac{1}{2}}} \mathrm{~d} X}{1+\frac{1}{2}(\gamma-1) \bar{M}_{2}^{2}\left(1-\frac{1}{2}(\gamma+1)\right)},
$$

with

$$
A_{3}=\bar{M}_{2}\left(1+\frac{1}{2}(\gamma-1) \bar{M}_{2}^{2}\right)^{\frac{1}{2}}, \quad A_{4}=-\frac{1}{4}\left(\gamma^{2}-1\right) \bar{M}_{2}\left(1+\frac{1}{2}(\gamma-1) \bar{M}_{2}^{2}\right)^{\frac{1}{2}}
$$

These effective lengths may be combined with the compact transmission coefficients obtained by Marble and Candel [11] to provide a prediction for both the magnitude and phase of the transmitted acoustics. Consider the straightwalled nozzle illustrated in Figure 1: the flow perturbations may be separated into planar waves in accordance with equations (2a) and (2b). Upstream of the abrupt nozzle throat, the incident wave travels a distance $\ell_{1}$. The transmitted acoustic waves propagate from the throat to the exit through the length $\ell_{2}$. Therefore, the response of a supercritical nozzle to entropy disturbances $\sigma$ may be expressed, to first order in $\Omega$, as

$$
\begin{aligned}
& \frac{P_{2}^{+}}{\sigma}=\left|\frac{P_{2}^{+}}{\sigma}\right| \mathrm{e}^{\mathrm{i} k_{2}^{+} \ell_{2, \sigma}+\mathrm{i} k_{0}^{+} \ell_{1}}+O\left(\Omega^{2}\right), \\
& \frac{P_{2}^{-}}{\sigma}=\left|\frac{P_{2}^{-}}{\sigma}\right| \mathrm{e}^{\mathrm{i} k_{2}^{-} \ell_{2, \sigma}+\mathrm{i} k_{0}^{+} \ell_{1}}+O\left(\Omega^{2}\right),
\end{aligned}
$$

where $k_{2}^{+}=\omega /\left(\bar{c}_{2}+\bar{u}_{2}\right), k_{2}^{-}=\omega /\left(\bar{c}_{2}-\bar{u}_{2}\right)$, and $k_{0}^{+}=\omega / \bar{u}_{1}$. The magnitude coefficients are the Marble and Candel compact expressions [11] (equations A.1 and A.2 in Appendix A). 
Similarly, the response to incident acoustic perturbations $P_{1}^{+}$may be written as

$$
\begin{aligned}
& \frac{P_{2}^{+}}{P_{1}^{+}}=\left|\frac{P_{2}^{+}}{P_{1}^{+}}\right| \mathrm{e}^{\mathrm{i} k_{2}^{+} \ell_{2, p}+\mathrm{i} k_{1}^{+} \ell_{1}}+O\left(\Omega^{2}\right), \\
& \frac{P_{2}^{-}}{P_{1}^{+}}=\left|\frac{P_{2}^{-}}{P_{1}^{+}}\right| \mathrm{e}^{\mathrm{i} k_{2}^{-} \ell_{2, p}+\mathrm{i} k_{1}^{+} \ell_{1}}+O\left(\Omega^{2}\right) .
\end{aligned}
$$

Here, $k_{1}^{+}=\omega /\left(\bar{c}_{1}+\bar{u}_{1}\right)$, and the compact coefficients are expressions A.3 and A.4.

\subsection{Comparison with numerical results}

The analytical predictions are compared against numerical results from a quasi one-dimensional simulation that solves the finite volume form of the inviscid equations described in Section 2. Originally developed by Denton [32], the code utilises a small amount of explicit artificial viscosity to suppress any numerical instability in the centred second-order, time-marching algorithm that updates the mass, momentum and energy fluxes across each cell until it converges onto a steady-flow solution in the time domain. A deferred correction technique is applied to mitigate non-physical dissipation effects, improving the accuracy of the solution. The mean Mach number through a typical supercritical nozzle, with inlet stagnation pressure and temperature of $216 \mathrm{kPa}$ and 950 $\mathrm{K}$, and downstream pressure of $41.5 \mathrm{kPa}$, is shown in Figure 2 .

The simulation consists of two codes. The first calculates the mean steady flow properties through the nozzle. The second code solves for the inviscid linearised perturbations for a specified range of frequencies, similar to that of Stow et al. [12]. The mean flow results are used as a base flow for this second calculation, where either an incident acoustic or entropy disturbance may be prescribed. To avoid an accumulation of acoustic energy, the outgoing and reflected plane waves at the nozzle inlet and exit are separated at each time step, and the reflected components removed, thereby ensuring that the boundaries of the computation domain are non-reflective.

The acoustic response of a supercritical nozzle is plotted in Figure 3 where a downstream-propagating acoustic disturbance has been prescribed at the nozzle inlet, and in Figure 4 with an entropy wave input. As expected, the Marble and Candel coefficients for the compact nozzle [11] provide reasonable predictions for the magnitude of the transmitted noise, particularly for low non-dimensional frequency. Both figures 3 and 4 also show that the numerical data for the phase response for low $\Omega /(2 \pi)$ agree well with the new analytical predictions (16) and (17), using $\ell_{2, p}$ and $\ell_{2, \sigma}$ respectively.

\section{The supercritical nozzle with shock}

Although the analysis performed in Section 3 assumed that the exit flow from the nozzle is supercritical and free from the presence of any shock waves, 


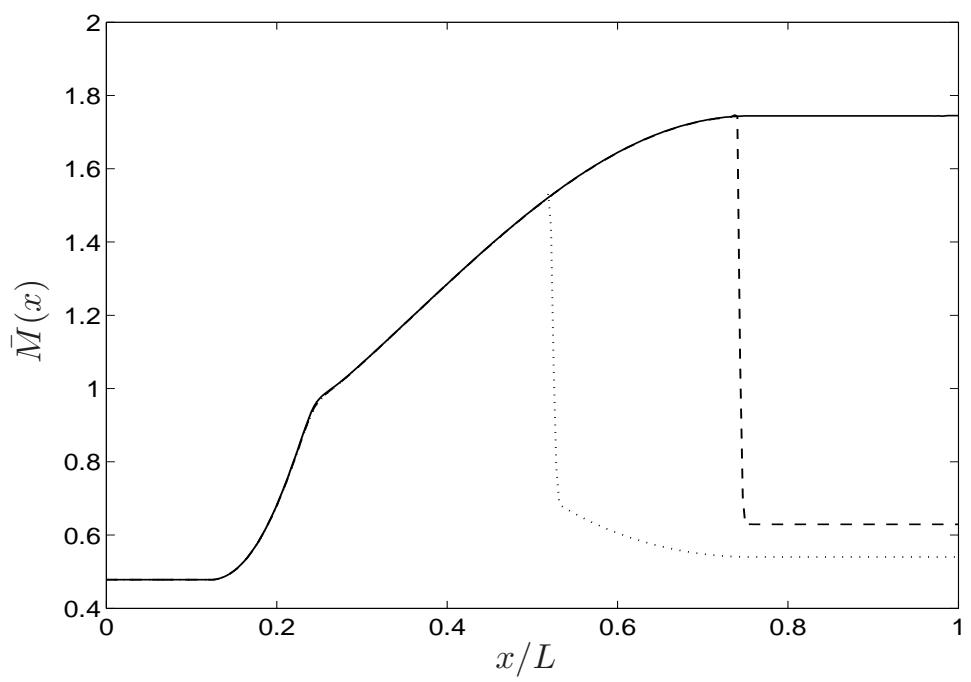

Figure 2: Mach number variation of the mean flow. Corresponding to cases for increasing downstream pressure, the supercritical nozzle in Section $3(-)$, shock just downstream of the divergent section in Section 4.1 (--), and shock in the diffuser in Section $4.2(\cdot \cdot)$, are shown.

(a)
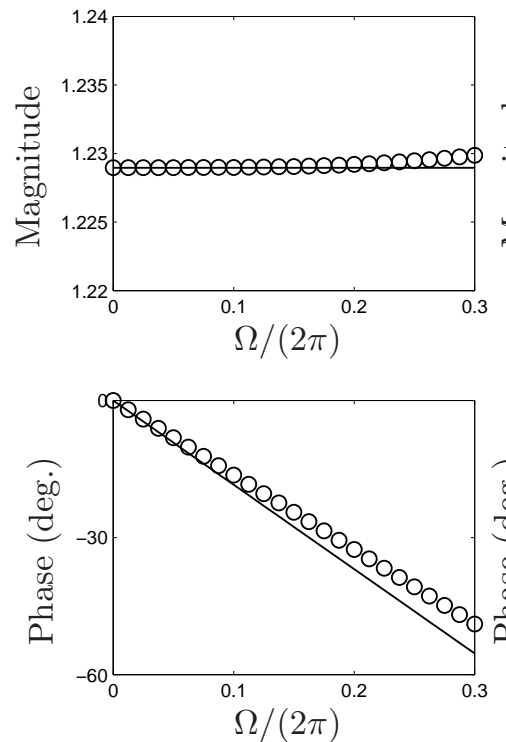

(b)
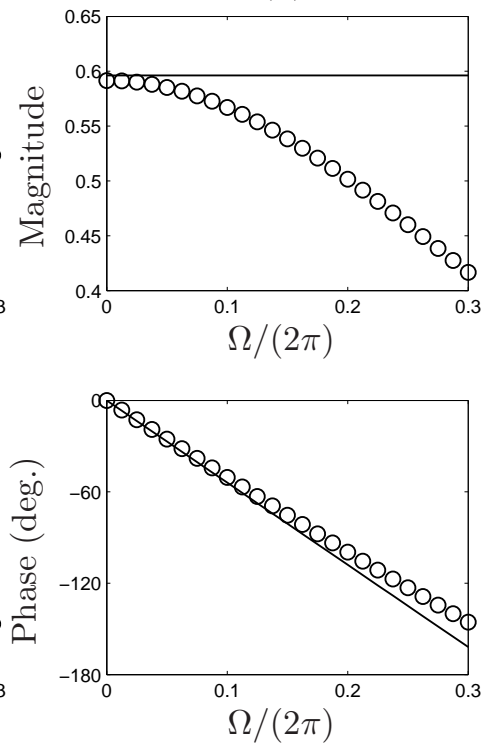

Figure 3: Magnitude and phase of the transmission coefficients, (a) $P_{2}^{+}$and (b) $P_{2}^{-}$, of a supercritical nozzle with an acoustic disturbance at the inlet. Analytical predictions are represented by $(-)$ and numerical results by $(\circ)$. 
(a)
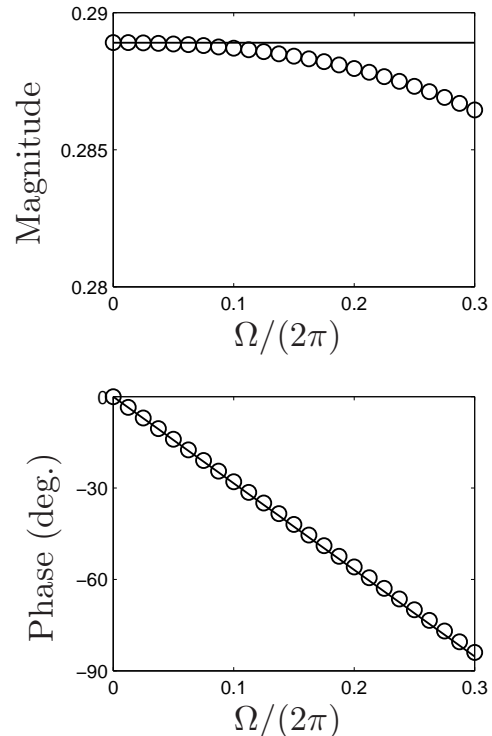

(b)
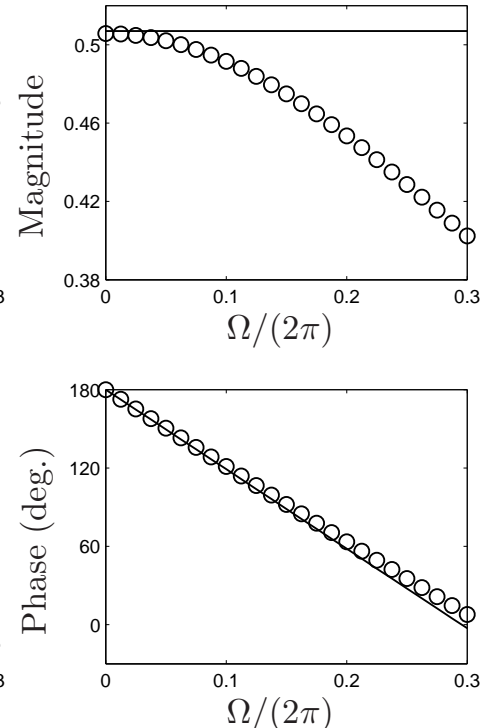

Figure 4: Magnitude and phase of the transmission coefficients, (a) $P_{2}^{+}$and (b) $P_{2}^{-}$, of a supercritical nozzle with an entropy disturbance at the inlet. Analytical predictions are represented by $(-)$ and numerical results by $(\circ)$.

it is perhaps more realistic to investigate the response of a nozzle with one. The flow properties across a normal shock, in the frame of reference where it is stationary (denoted by 'sh'), is given by the Rankine-Hugoniot relations,

$$
\begin{gathered}
\frac{u_{3, \mathrm{sh}}}{u_{2, \mathrm{sh}}}=\frac{1+\frac{1}{2}(\gamma-1) M_{2, \mathrm{sh}}^{2}}{\frac{1}{2}(\gamma+1) M_{2, \mathrm{sh}}^{2}}, \quad \frac{p_{3, \mathrm{sh}}}{p_{2, \mathrm{sh}}}=\frac{\gamma M_{2, \mathrm{sh}}^{2}-\frac{1}{2}(\gamma-1)}{\frac{1}{2}(\gamma+1)}, \\
\frac{\rho_{3, \mathrm{sh}}}{\rho_{2, \mathrm{sh}}}=\frac{\frac{1}{2}(\gamma+1) M_{2, \mathrm{sh}}^{2}}{1+\frac{1}{2}(\gamma-1) M_{2, \mathrm{sh}}^{2}},
\end{gathered}
$$

where 2 and 3 indicate the positions just upstream and downstream of the shock respectively.

\subsection{Shock downstream of nozzle}

Consider first the less complex case of a nozzle with a planar shock located just downstream of the divergent section of the nozzle, where the duct is straight. The shock wave has the velocity $u_{s}^{\prime}$ relative to its mean position. Therefore,

$$
M_{2, \mathrm{sh}}=\bar{M}_{2}\left(1+\frac{u_{2}^{\prime}}{\bar{u}_{2}}-\frac{c_{2}^{\prime}}{\bar{c}_{2}}-\frac{u_{s}^{\prime}}{\bar{u}_{2}}\right) .
$$


Neglecting the second-order products of perturbation quantities,

$$
\frac{c^{\prime}}{\bar{c}}=\frac{1}{2}\left(\frac{p^{\prime}}{\bar{p}}-\frac{\rho^{\prime}}{\bar{\rho}}\right) .
$$

Hence,

$$
\begin{aligned}
M_{2, \mathrm{sh}} & =\bar{M}_{2}\left(1+\frac{M_{2}^{\prime}}{\bar{M}_{2}}-\frac{u_{s}^{\prime}}{\bar{u}_{2}}\right), \\
\frac{M_{2}^{\prime}}{\bar{M}_{2}} & =\frac{u_{2}^{\prime}}{\bar{u}_{2}}+\frac{1}{2} \frac{\rho_{2}^{\prime}}{\bar{\rho}_{2}}-\frac{\gamma}{2} \frac{p_{2}^{\prime}}{\gamma \bar{p}_{2}} .
\end{aligned}
$$

Substituting equation (18) into the Rankine-Hugoniot relations and linearising leads to

$$
\begin{aligned}
& \frac{\bar{u}_{3}}{\bar{u}_{2}}\left(\frac{u_{3}^{\prime}}{\bar{u}_{3}}-\frac{u_{2}^{\prime}}{\bar{u}_{2}}\right)=\left(\frac{4}{(\gamma+1) \bar{M}_{2}^{2}}-\frac{\bar{u}_{3}}{\bar{u}_{2}}+1\right) \frac{u_{s}^{\prime}}{\bar{u}_{2}}-\frac{4}{(\gamma+1) \bar{M}_{2}^{2}} \frac{M_{2}^{\prime}}{\bar{M}_{2}}, \\
& \frac{\bar{p}_{3}}{\bar{p}_{2}}\left(\frac{p_{3}^{\prime}}{\bar{p}_{3}}-\frac{p_{2}^{\prime}}{\bar{p}_{2}}\right)=\frac{4 \gamma \bar{M}_{2}^{2}}{(\gamma+1)}\left(\frac{M_{2}^{\prime}}{\bar{M}_{2}}-\frac{u_{s}^{\prime}}{\bar{u}_{2}}\right), \\
& \frac{\bar{\rho}_{3}}{\bar{\rho}_{2}}\left(\frac{\rho_{3}^{\prime}}{\bar{\rho}_{3}}-\frac{\rho_{2}^{\prime}}{\bar{\rho}_{2}}\right)=\frac{(\gamma+1) \bar{M}_{2}^{2}}{\left[1+\frac{1}{2}(\gamma-1) \bar{M}_{2}^{2}\right]^{2}}\left(\frac{M_{2}^{\prime}}{\bar{M}_{2}}-\frac{u_{s}^{\prime}}{\bar{u}_{2}}\right) .
\end{aligned}
$$

To investigate the acoustic response downstream of the shock, $u_{s}^{\prime}$ may be eliminated between equations (20) and (21), giving

$$
\begin{aligned}
2 \bar{M}_{2}^{2} \bar{M}_{3}^{2}\left(\frac{u_{3}^{\prime}}{\bar{u}_{3}}\right)+\left(1+\bar{M}_{2}^{2}\right)\left(\frac{p_{3}^{\prime}}{\gamma \bar{p}_{3}}\right)= & \\
& 2 \bar{M}_{2}^{2} \bar{M}_{3}^{2}\left(\frac{u_{2}^{\prime}}{\bar{u}_{2}}\right)+\left(1+\bar{M}_{2}^{2}\right)\left(\frac{p_{2}^{\prime}}{\gamma \bar{p}_{2}}\right)+\frac{2 \bar{M}_{2}^{2}\left(\bar{M}_{2}^{2}-1\right)}{\gamma \bar{M}_{2}^{2}-\frac{1}{2}(\gamma-1)} \frac{M_{2}^{\prime}}{\bar{M}_{2}}
\end{aligned}
$$

Following the methods used in the previous section, the perturbation quantities may be expanded in terms of $\Omega$; and to zeroth order, equation (19) implies that $M_{2}^{\prime}=0$ due to the boundary condition given by the choked nozzle in relation (8). This corresponds to Marble and Candel's assumption that the fluctuating component in Mach number just upstream of the normal shock can be ignored when the nozzle is treated as compact [11].

When terms to higher orders of $\Omega$ are not neglected, and remembering that $\sigma=p^{\prime} /(\gamma \bar{p})-\rho^{\prime} / \bar{\rho}$, equation (23) may be written to express the acoustic wave downstream of the shock, $P_{3}^{+}$, in terms of the planar acoustic and entropy waves upstream of the shock, $P_{2}^{+}, P_{2}^{-}$and $\sigma_{2}$. If the nozzle exit is anechoic, $P_{3}^{-}=0$. These waves are vectors, having both magnitude and phase.

$$
P_{3}^{+}=\alpha^{+} P_{2}^{+}+\alpha^{-} P_{2}^{-}-\beta \sigma_{2},
$$


where the transmission factors are

$$
\begin{aligned}
\alpha^{+} & =\frac{1+\bar{M}_{2}^{2}+2 \bar{M}_{2} \bar{M}_{3}^{2}}{1+\bar{M}_{2}^{2}+2 \bar{M}_{2}^{2} \bar{M}_{3}}+\frac{\bar{M}_{2}\left(\bar{M}_{2}^{2}-1\right)\left[2-(\gamma-1) \bar{M}_{2}\right]}{\left[1+\bar{M}_{2}^{2}+2 \bar{M}_{2}^{2} \bar{M}_{3}\right]\left[\gamma \bar{M}_{2}^{2}-\frac{1}{2}(\gamma-1)\right]}, \\
\alpha^{-} & =\frac{1+\bar{M}_{2}^{2}-2 \bar{M}_{2} \bar{M}_{3}^{2}}{1+\bar{M}_{2}^{2}+2 \bar{M}_{2}^{2} \bar{M}_{3}}-\frac{\bar{M}_{2}\left(\bar{M}_{2}^{2}-1\right)\left[2+(\gamma-1) \bar{M}_{2}\right]}{\left[1+\bar{M}_{2}^{2}+2 \bar{M}_{2}^{2} \bar{M}_{3}\right]\left[\gamma \bar{M}_{2}^{2}-\frac{1}{2}(\gamma-1)\right]}, \\
\beta & =\frac{\bar{M}_{2}^{2}\left(\bar{M}_{2}^{2}-1\right)}{\left[1+\bar{M}_{2}^{2}+2 \bar{M}_{2}^{2} \bar{M}_{3}\right]\left[\gamma \bar{M}_{2}^{2}-\frac{1}{2}(\gamma-1)\right]} .
\end{aligned}
$$

Equation (24) represents a revised version of the Rankine-Hugoniot relationship for linearised perturbations across a normal shock wave. The frequencydependent phase of the incident waves just upstream of the shock, $P_{2}^{+}, P_{2}^{-}$and $\sigma_{2}$, may be analytically derived using the effective lengths suggested in Section 3.1. It has been assumed that there is no dissipation or dispersion of the entropy wave, which has been advected through $\ell_{1}$ and $\ell_{2, \sigma}$;

$$
\sigma_{2}=|\sigma| \mathrm{e}^{\mathrm{i}\left(\omega / \bar{u}_{1}\right) \ell_{1}+\mathrm{i}\left(\omega / \bar{u}_{2}\right) \ell_{2, \sigma}}
$$

Likewise, equations (21) and (22) provide an expression for the entropy disturbance generated by the oscillating shock wave:

$$
\sigma_{3}=\psi \alpha^{+} P_{2}^{+}+\psi \alpha^{-} P_{2}^{-}+(1-\psi \beta) \sigma_{2},
$$

where

$$
\psi=1-\frac{\gamma \bar{M}_{2}^{2}-\frac{1}{2}(\gamma-1)}{\bar{M}_{2}^{2}\left[1+\frac{1}{2}(\gamma-1) \bar{M}_{2}^{2}\right]} .
$$

The transmission factors associated with $P_{2}^{+}, P_{2}^{-}$and $\sigma_{2}$ in equations (24) and (26) have been plotted against $\bar{M}_{2}$ in Figure 5 , where the variance from Marble and Candel's analytical results [11] can be seen.

For the supercritical nozzle without the presence of a shock in the previous section, the analytical prediction for the magnitude of the transmitted noise did not vary with frequency. Nonetheless, the model was a good approximation at low $\Omega$. The acoustic, and entropy, response after a shock downstream of a nozzle as expressed in equations (24) and (26) are, however, combinations of vectors whose phase angles vary with frequency. Hence, both magnitude and phase predictions are not fixed to their 'compact' values. These results were compared against a numerical simulation, using the codes described in Section 3.2 , and were found to be a good model. Figures 6 and 7 show the acoustic response downstream of the shock, $P_{3}^{+}$, caused, respectively, by incident entropy or acoustic waves at the inlet (results for low $\Omega$ have been omitted in Figure 7 due to some numerical difficulties encountered).

\subsection{Shock in nozzle divergence}

In general, a normal shock may occur in the divergent section of the nozzle. The linearised interaction between the flow perturbations and the moving shock 

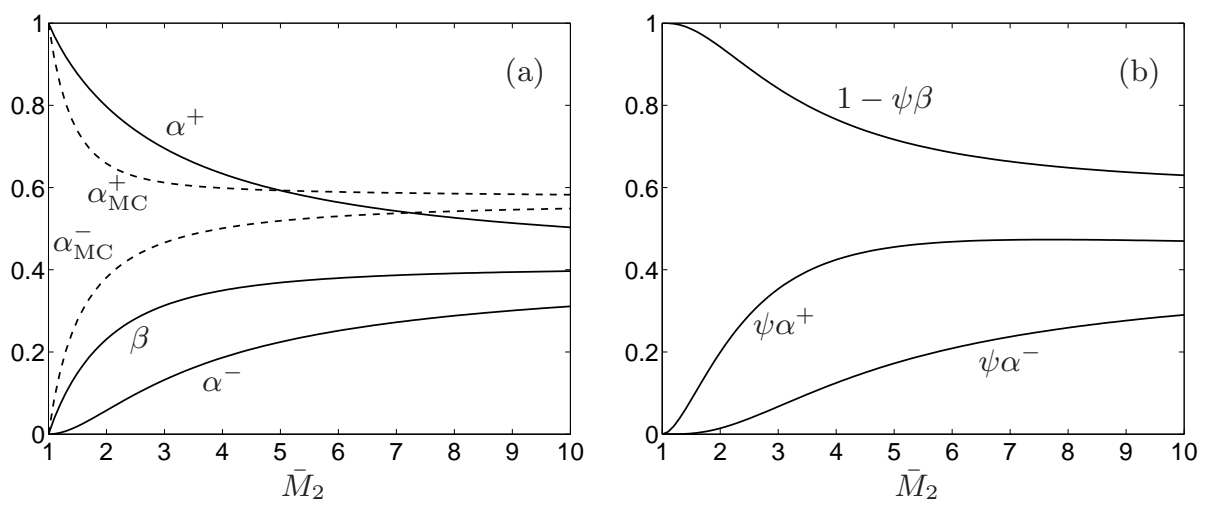

Figure 5: Transmission factors of (a) the acoustic response, $P_{3}^{+}$, and (b) entropy response, $\sigma_{3}$, downstream of a shock in the straight section of the nozzle, as expressed in equations (24) and (26). The factors are plotted as a function of the shock strength, $\bar{M}_{2}$. The subscript 'MC' denotes Marble and Candel's results [11].
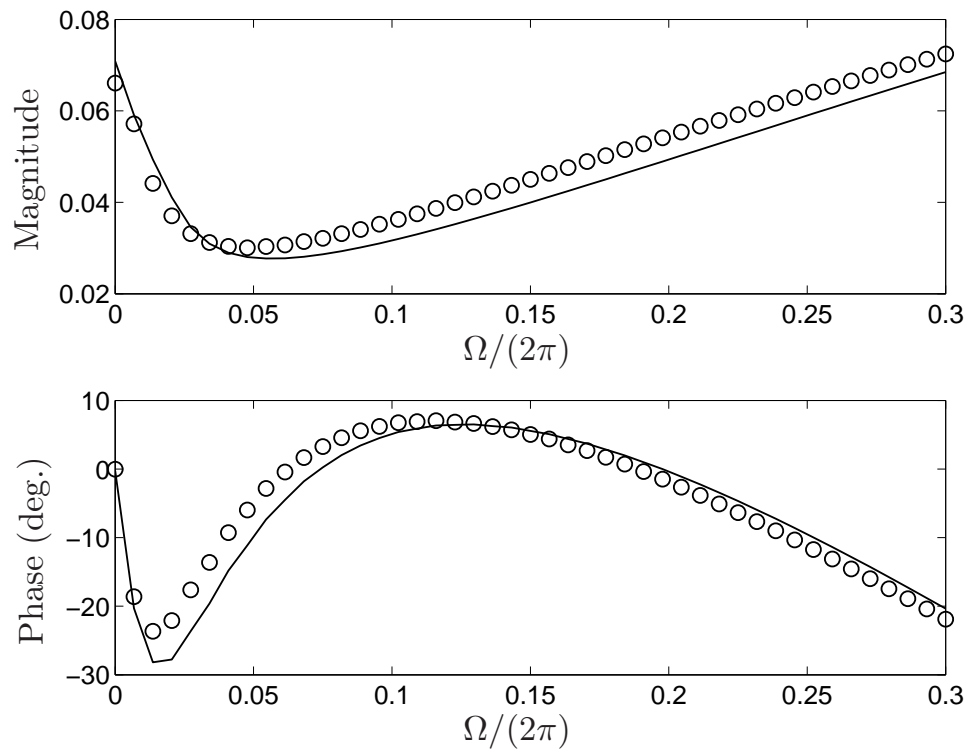

Figure 6: Magnitude and phase of $P_{3}^{+}$from a supercritical nozzle with shock downstream of the divergence and an entropy disturbance at the inlet. Analytical predictions are represented by $(-)$ and numerical results by $(\circ)$. 

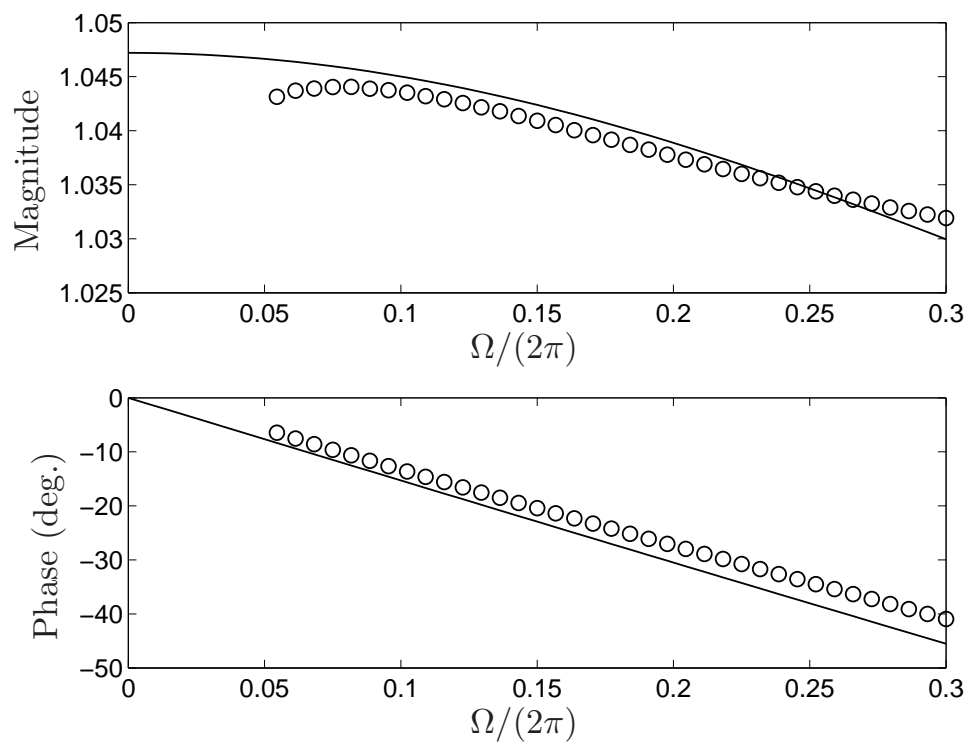

Figure 7: Magnitude and phase of $P_{3}^{+}$from a supercritical nozzle with shock downstream of the divergence and an acoustic disturbance at the inlet. Analytical predictions are represented by $(-)$ and numerical results by $(\circ)$.

wave has been studied by Kuo and Dowling [15], and Stow et al. [12] when the fluctuations upstream of the shock may be neglected. The analysis was revisited by Moase et al. [13] for when the assumption of negligible upstream disturbances is no longer valid. A similar derivation leads to equations for the pressure, velocity and density fluctuations just downstream of the shock:

$$
\begin{aligned}
\frac{p_{3}^{\prime}}{\gamma \bar{p}_{3}} & =\frac{x_{s}^{\prime}}{\bar{M}_{2}\left[1+\frac{1}{2}(\gamma-1) \bar{M}_{2}^{2}\right]} F_{p}+\frac{p_{2}^{\prime}}{\gamma \bar{p}_{2}}+\frac{2 \bar{M}_{2}^{2}}{\gamma \bar{M}_{2}^{2}-\frac{1}{2}(\gamma-1)} \frac{M_{2}^{\prime}}{\bar{M}_{2}}, \\
\frac{u_{3}^{\prime}}{\bar{u}_{3}} & =-\frac{x_{s}^{\prime}}{\bar{M}_{2}\left[1+\frac{1}{2}(\gamma-1) \bar{M}_{2}^{2}\right]} F_{u}+\frac{u_{2}^{\prime}}{\bar{u}_{2}}-\frac{2}{1+\frac{1}{2}(\gamma-1) \bar{M}_{2}^{2}} \frac{M_{2}^{\prime}}{\bar{M}_{2}}, \\
\frac{\rho_{3}^{\prime}}{\bar{\rho}_{3}} & =\frac{x_{s}^{\prime}}{\bar{M}_{2}\left[1+\frac{1}{2}(\gamma-1) \bar{M}_{2}^{2}\right]} F_{\rho}+\frac{\rho_{2}^{\prime}}{\bar{\rho}_{2}}+\frac{2}{1+\frac{1}{2}(\gamma-1) \bar{M}_{2}^{2}} \frac{M_{2}^{\prime}}{\bar{M}_{2}} .
\end{aligned}
$$

The analysis, as well as the expressions for $F_{p}, F_{u}$ and $F_{\rho}$, are outlined in Appendix B. $x_{s}^{\prime}$ is the fluctuating term for the shock position. Taking the velocity distribution through the nozzle to be piece-wise linear, Moase et al. [13] used these results in conjunction with Tsien's solution to the hyper-geometric problem [33] to formulate matching conditions between the linear velocity sections of the nozzle. This enabled the prediction of both the acoustic magnitude and phase from finite-length nozzles.

This section, however, describes an alternative method that does not require the velocity through the nozzle to be piece-wise linear, which avoids the cum- 


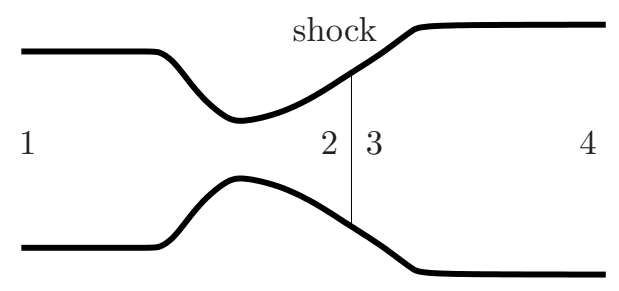

Figure 8: Sketch of supercritical nozzle with a shock in the divergent section. The numbers correspond to the location subscripts.

bersome hyper-geometric equations and the matching conditions necessary to solve them. The results are valid for low $\Omega$, when combustion noise is significant. Unlike the case in Section 4.1, the pressure perturbation just downstream of the shock consists of a downstream-propagating $P_{3}^{+}$as well as the upstreamtravelling $P_{3}^{-}$. The deceleration of the mean flow in the subsonic diffuser section between the shock position and the nozzle exit generates $P_{3}^{-}$through its interaction with $P_{3}^{+}$and the entropy wave $\sigma_{3}$ caused by the shock oscillations. Hence, in order to predict the transmitted noise at the exit $P_{4}^{+}$, four equations are required to solve for the four unknowns. (The subscript numbers correspond to the locations indicated in Figure 8.)

The first two equations may be obtained by eliminating $x_{s}^{\prime}$ between equations (27) and (28), and between (27) and (29), leading to

$$
\begin{aligned}
& \frac{u_{3}^{\prime}}{\bar{u}_{3}}+\frac{F_{u}}{F_{p}} \frac{p_{3}^{\prime}}{\gamma \bar{p}_{3}}=\frac{F_{u}}{F_{p}} \frac{p_{2}^{\prime}}{\gamma \bar{p}_{2}}+\frac{u_{2}^{\prime}}{\bar{u}_{2}}+\Phi \frac{M_{2}^{\prime}}{\bar{M}_{2}}, \\
& \frac{\rho_{3}^{\prime}}{\bar{\rho}_{3}}-\frac{F_{\rho}}{F_{p}} \frac{p_{3}^{\prime}}{\gamma \bar{p}_{3}}=-\frac{F_{\rho}}{F_{p}} \frac{p_{2}^{\prime}}{\gamma \bar{p}_{2}}+\frac{\rho_{2}^{\prime}}{\bar{\rho}_{2}}+\Psi \frac{M_{2}^{\prime}}{\bar{M}_{2}} .
\end{aligned}
$$

where

$$
\begin{aligned}
& \Phi=\frac{F_{u}}{F_{p}} \frac{2 \bar{M}_{2}^{2}}{\gamma \bar{M}_{2}^{2}-\frac{1}{2}(\gamma-1)}-\frac{2}{1+\frac{1}{2}(\gamma-1) \bar{M}_{2}^{2}}, \\
& \Psi=\frac{2}{1+\frac{1}{2}(\gamma-1) \bar{M}_{2}^{2}}-\frac{F_{\rho}}{F_{p}} \frac{2 \bar{M}_{2}^{2}}{\gamma \bar{M}_{2}^{2}-\frac{1}{2}(\gamma-1)} .
\end{aligned}
$$

The acoustic perturbations in equation (30) may be rewritten as planar acoustic waves, as expressed by equations (2a) and (2b). This is only valid locally as the cross-sectional area is changing with axial distance;

$$
\begin{aligned}
P_{3}^{+}\left[\frac{F_{u}}{F_{p}}+\frac{1}{\bar{M}_{3}}\right]+P_{3}^{-}\left[\frac{F_{u}}{F_{p}}\right. & \left.-\frac{1}{\bar{M}_{3}}\right]=P_{2}^{+}\left[\frac{F_{u}}{F_{p}}-\frac{1}{2}(\gamma-1) \Phi+\frac{1}{\bar{M}_{2}}+\frac{\Phi}{\bar{M}_{2}}\right] \\
& +P_{2}^{-}\left[\frac{F_{u}}{F_{p}}-\frac{1}{2}(\gamma-1) \Phi-\frac{1}{\bar{M}_{2}}-\frac{\Phi}{\bar{M}_{2}}\right]-\frac{\Phi}{2} \sigma_{2} .
\end{aligned}
$$


Remembering that the density perturbation may be expressed in terms of pressure and entropy fluctuations, equation (31) may be similarly rearranged as

$$
\begin{aligned}
P_{3}^{+}\left[1-\frac{F_{\rho}}{F_{p}}\right]+P_{3}^{-} & {\left[1-\frac{F_{\rho}}{F_{p}}\right]-\sigma_{3}=P_{2}^{+}\left[1-\frac{F_{\rho}}{F_{p}}-\frac{1}{2}(\gamma-1) \Psi+\frac{\Psi}{\bar{M}_{2}}\right] } \\
& +P_{2}^{-}\left[1-\frac{F_{\rho}}{F_{p}}-\frac{1}{2}(\gamma-1) \Psi-\frac{\Psi}{\bar{M}_{2}}\right]-\left(1+\frac{\Psi}{2}\right) \sigma_{2} .
\end{aligned}
$$

Note that the right-hand sides of equations (32) and (33), or $\mathrm{RHS}_{\Phi}$ and $\mathrm{RHS}_{\Psi}$, are known quantities.

The final two equations may be obtained by considering Marble and Candel's compact expressions for the acoustic reflection and transmission from a subcritical diffuser (Appendix A), together with effective lengths to allow for frequency-dependence. If the resulting complex coefficients may be expressed as $\zeta$, the linear combination of the incident acoustic and entropy waves produce

$$
\begin{aligned}
& P_{3}^{-}=\zeta_{\sigma}^{-} \sigma_{3}+\zeta_{p}^{-} P_{3}^{+}, \\
& P_{4}^{+}=\zeta_{\sigma}^{+} \sigma_{3}+\zeta_{p}^{+} P_{3}^{+},
\end{aligned}
$$

where the abrupt change in area of the equivalent diffuser has been assumed to have occurred at the position where $\bar{M}=\left(\bar{M}_{3}+\bar{M}_{4}\right) / 2$.

The four equations (32)-(35) may be expressed in a matrix form,

$$
\left(\begin{array}{cccc}
\frac{F_{u}}{F_{p}}+\frac{1}{M_{3}} & \frac{F_{u}}{F_{p}}-\frac{1}{M_{3}} & 0 & 0 \\
1-\frac{F_{\rho}}{F_{p}} & 1-\frac{F_{\rho}}{F_{p}} & -1 & 0 \\
-\zeta_{p}^{-} & 1 & -\zeta_{\sigma}^{-} & 0 \\
-\zeta_{p}^{+} & 0 & -\zeta_{\sigma}^{+} & 1
\end{array}\right)\left[\begin{array}{c}
P_{3}^{+} \\
P_{3}^{-} \\
\sigma_{3} \\
P_{4}^{+}
\end{array}\right]=\left(\begin{array}{c}
\mathrm{RHS}_{\Phi} \\
\mathrm{RHS}_{\Psi} \\
0 \\
0
\end{array}\right)
$$

in order to solve for $P_{4}^{+}$. The numerical technique used in Section 3.2 was used to simulate the case when a shock exists in the divergent section of a convergentdivergent nozzle. The mean Mach number through the nozzle is given in Figure 2. As shown in figures 9 and 10 , the analytical $P_{4}^{+}$gives a good prediction for both the magnitude and phase of the calculated transmitted noise.

\section{Conclusions}

An asymptotic analysis of the linearised flow equations led to the formulation of effective lengths used in the phase prediction of the response of supercritical nozzles. These length expressions were found to be dependent on the type of incident disturbance, and were only a function of the Mach number variation through the nozzle. The resulting phase predictions are valid for low nondimensional frequency perturbations, when the nozzle is choked.

The analysis was also extended to the case where a normal shock wave is present downstream of the divergence section of the nozzle. Just upstream of 

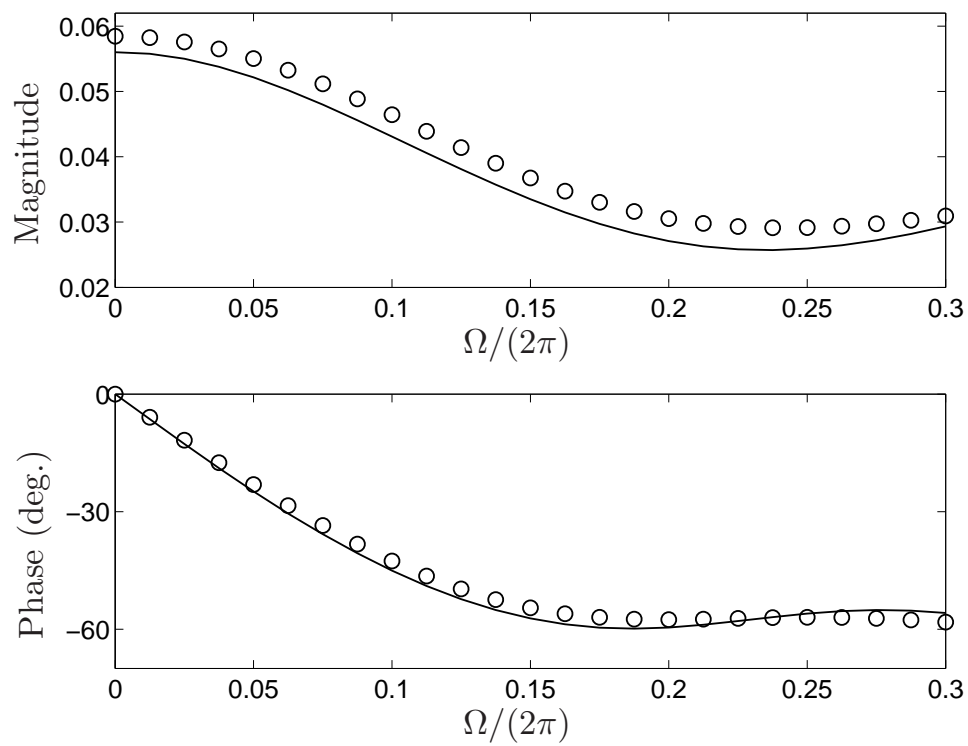

Figure 9: Magnitude and phase of $P_{4}^{+}$from a supercritical nozzle with shock in the divergence and an entropy disturbance at the inlet. Analytical predictions are represented by $(-)$ and numerical results by $(\circ)$.
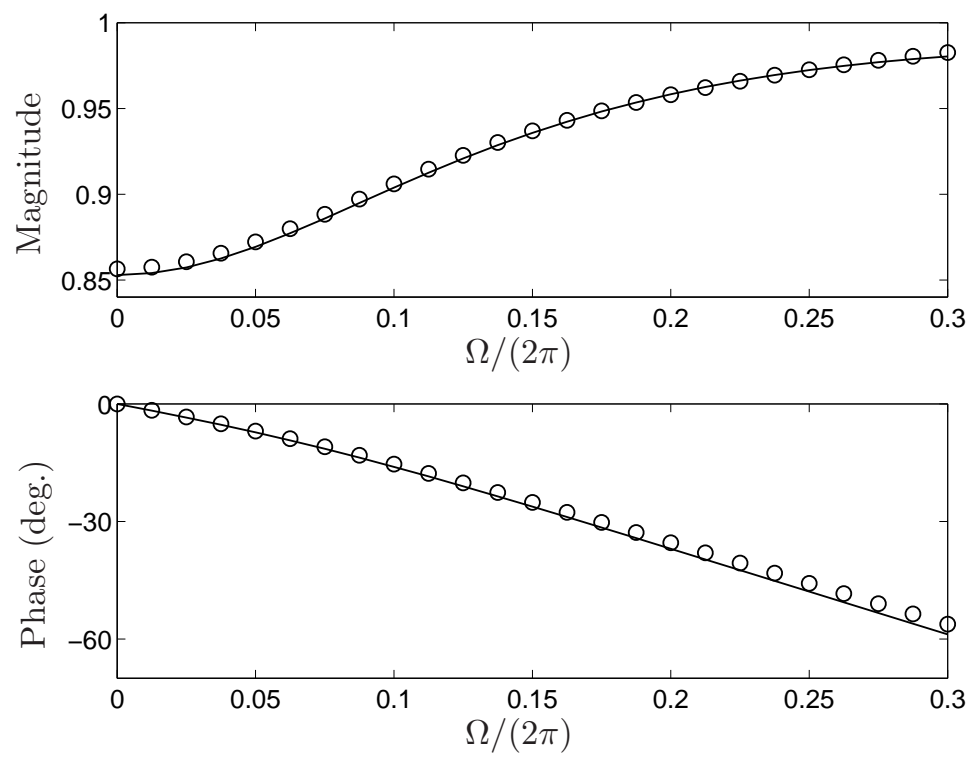

Figure 10: Magnitude and phase of $P_{4}^{+}$from a supercritical nozzle with shock in the divergence and an acoustic disturbance at the inlet. Analytical predictions are represented by (-) and numerical results by (o). 


\begin{tabular}{ll}
\hline Case & Method \\
\hline \hline $\begin{array}{l}\text { Response of supercritical nozzle } \\
\text { to entropy disturbance }\end{array}$ & $\begin{array}{l}\text { Obtain } P_{2}^{+} \text {and } P_{2}^{-} \text {from equations (16) with } \\
\ell_{1} \text { from }(10) \text { and } \ell_{2, \sigma} \text { from }(14)\end{array}$ \\
\hline $\begin{array}{l}\text { Response of supercritical nozzle } \\
\text { to acoustic disturbance }\end{array}$ & $\begin{array}{l}\text { Obtain } P_{2}^{+} \text {and } P_{2}^{-} \text {from equations }(17) \text { with } \\
\ell_{1} \text { from }(10) \text { and } \ell_{2, p} \text { from }(15)\end{array}$ \\
\hline $\begin{array}{l}\text { Response of nozzle with normal } \\
\text { shock after nozzle divergence to }\end{array}$ & $\begin{array}{l}\text { Obtain } P_{2}^{+} \text {and } P_{2}^{-} \text {from equations }(16), \sigma_{2} \\
\text { entropy disturbance } \\
P_{3}^{+}\end{array}$ \\
\hline $\begin{array}{l}\text { Response of nozzle with normal } \\
\text { shock after nozzle divergence to }\end{array}$ & $\begin{array}{l}\text { Obtain } P_{2}^{+} \text {and } P_{2}^{-} \text {from equation }(24) \text { to obtain } \\
\text { acoustic disturbance }\end{array}$ \\
\hline $\begin{array}{l}\text { Response of nozzle with normal } \\
\text { shock in nozzle divergence }\end{array}$ & $\begin{array}{l}\text { Obtain } P_{2}^{+} \text {and } P_{2}^{-} \text {from equations }(16) \\
\text { and/or }(17), \sigma_{2} \text { from }(25), \text { then solve matrix }\end{array}$ \\
\hline
\end{tabular}

Table 1: Summary of analytical expressions for transmitted combustion noise.

the shock the flow perturbations may be separated into two acoustic waves propagating at different speeds, and an entropy wave. The acoustic response after the shock was found to be a linear combination of these perturbations, governed by the linearised Rankine-Hugoniot relations. When the shock exists within the divergence section, the phase response may be predicted by treating the nozzle as two parts; a supercritical nozzle up to the mean shock position and a subcritical diffuser section. It has been shown that there is reasonable agreement between the analytical predictions and numerical calculations, particularly for low $\Omega$. These results may be treated as an alternative method to that developed by Moase et al. [13] to predict the acoustic response for a choked nozzle.

The results for the various flow configurations considered in this paper are presented in Table 1. Although the nozzle responses to acoustic and entropy disturbances were treated separately, the total direct and indirect noise downstream of a nozzle may be considered as a superposition of the two cases. The phase prediction methods derived in this work may be validly applied to network or matrix thermoacoustic methods that track the noise through turbine stages (potentially using actuator disc theory for blade rows).

\section{Acknowledgements}

Chee Su Goh gratefully acknowledges the funding received from the Overseas Research Scholarship and from the Imperial College Student Opportunities Fund. 


\section{Appendix A. Compact nozzle coefficients}

The magnitudes of the transmission and reflection coefficients for a compact nozzle were analytically derived by Marble and Candel [11]. For the supercritical nozzle, referred to in Section 3.1, the transmission coefficients for an entropy disturbance input, $\sigma$, at the nozzle inlet are

$$
\begin{aligned}
& \left|\frac{P_{2}^{+}}{\sigma}\right|=\frac{1}{2}\left(\frac{\bar{M}_{2}-\bar{M}_{1}}{2}\right)\left[\frac{1}{1+\frac{1}{2}(\gamma-1) \bar{M}_{1}}\right], \\
& \left|\frac{P_{2}^{-}}{\sigma}\right|=-\frac{1}{2}\left(\frac{\bar{M}_{2}+\bar{M}_{1}}{2}\right)\left[\frac{1}{1+\frac{1}{2}(\gamma-1) \bar{M}_{1}}\right] .
\end{aligned}
$$

Likewise, when the nozzle has an acoustic disturbance upstream, $P_{1}^{+}$,

$$
\begin{aligned}
& \left|\frac{P_{2}^{+}}{P_{1}^{+}}\right|=\frac{1+\frac{1}{2}(\gamma-1) \bar{M}_{2}}{1+\frac{1}{2}(\gamma-1) \bar{M}_{1}}, \\
& \left|\frac{P_{2}^{-}}{P_{1}^{+}}\right|=\frac{1-\frac{1}{2}(\gamma-1) \bar{M}_{2}}{1+\frac{1}{2}(\gamma-1) \bar{M}_{1}} .
\end{aligned}
$$

For a subcritical nozzle or diffuser, where the flow is purely subsonic, the magnitude of the transmitted and reflected acoustic responses to entropy disturbances are

$$
\begin{aligned}
& \left|\zeta_{\sigma}^{+}\right|=\left|\frac{P_{4}^{+}}{\sigma}\right|=\left(\frac{\bar{M}_{4}-\bar{M}_{3}}{1+\bar{M}_{4}}\right)\left[\frac{\frac{1}{2} \bar{M}_{4}}{1+\frac{1}{2}(\gamma-1) \bar{M}_{3} \bar{M}_{4}}\right], \\
& \left|\zeta_{\sigma}^{-}\right|=\left|\frac{P_{3}^{-}}{\sigma}\right|=-\left(\frac{\bar{M}_{4}-\bar{M}_{3}}{1-\bar{M}_{3}}\right)\left[\frac{\frac{1}{2} \bar{M}_{3}}{1+\frac{1}{2}(\gamma-1) \bar{M}_{3} \bar{M}_{4}}\right] .
\end{aligned}
$$

When the disturbance is an upstream acoustic wave,

$$
\begin{aligned}
& \left|\zeta_{p}^{+}\right|=\left|\frac{P_{4}^{+}}{P_{3}^{+}}\right|=\left(\frac{2 \bar{M}_{4}}{1+\bar{M}_{4}}\right)\left(\frac{1+\bar{M}_{3}}{\bar{M}_{4}+\bar{M}_{3}}\right)\left[\frac{1+\frac{1}{2}(\gamma-1) \bar{M}_{4}^{2}}{1+\frac{1}{2}(\gamma-1) \bar{M}_{3} \bar{M}_{4}}\right], \\
& \left|\zeta_{p}^{-}\right|=\left|\frac{P_{3}^{-}}{P_{3}^{+}}\right|=\left(\frac{\bar{M}_{4}-\bar{M}_{3}}{1-\bar{M}_{3}}\right)\left(\frac{1+\bar{M}_{3}}{\bar{M}_{4}+\bar{M}_{3}}\right)\left[\frac{1-\frac{1}{2}(\gamma-1) \bar{M}_{3} \bar{M}_{4}}{1+\frac{1}{2}(\gamma-1) \bar{M}_{3} \bar{M}_{4}}\right] .
\end{aligned}
$$

The latter four expressions were used in Section 4.2.

\section{Appendix B. Dynamic shock equations}

The Rankine-Hugoniot relations state that across a normal shock,

$$
\frac{p_{3, \mathrm{sh}}}{p_{2, \mathrm{sh}}}=\frac{\gamma M_{2, \mathrm{sh}}^{2}-\frac{1}{2}(\gamma-1)}{\frac{1}{2}(\gamma+1)} .
$$


In the frame of reference following the moving shock,

$$
\begin{aligned}
M_{2, \mathrm{sh}} & =\bar{M}_{2}+M_{2}^{\prime}+x_{s}^{\prime}\left(\frac{\mathrm{d} \bar{M}_{2}}{\mathrm{~d} x}-\frac{\mathrm{i} \omega}{\bar{c}_{2}}\right), \\
p_{2, \mathrm{sh}} & =\bar{p}_{2}+p_{2}^{\prime}+x_{s}^{\prime} \frac{\mathrm{d} \bar{p}_{2}}{\mathrm{~d} x},
\end{aligned}
$$

given that the disturbance in the shock position has the form $x_{s}^{\prime}=\left|x_{s}^{\prime}\right| \mathrm{e}^{\mathrm{i} \omega t}$. From the ratio between static and stagnation pressures, we know that

$$
\frac{\mathrm{d} p}{\mathrm{~d} x}=-\frac{\gamma M p}{1+\frac{1}{2}(\gamma-1) M^{2}} \frac{\mathrm{d} M}{\mathrm{~d} x} .
$$

Applying equations (B.3) and (B.2) to (B.1) and linearising;

$$
\begin{array}{r}
\frac{p_{3, \mathrm{sh}}}{\bar{p}_{3}} \simeq 1+\gamma \bar{M}_{2} x_{s}^{\prime}\left\{\frac{\mathrm{d} \bar{M}_{2}}{\mathrm{~d} x}\left[\frac{2}{\gamma \bar{M}_{2}^{2}-\frac{1}{2}(\gamma-1)}-\frac{1}{1+\frac{1}{2}(\gamma-1) \bar{M}_{2}^{2}}\right]\right. \\
\left.-\frac{2}{\gamma \bar{M}_{2}^{2}-\frac{1}{2}(\gamma-1)} \frac{\mathrm{i} \omega}{\bar{c}_{2}}\right\}+\frac{p_{2}^{\prime}}{\bar{p}_{2}}+\frac{2 \gamma \bar{M}_{2}^{2}}{\gamma \bar{M}_{2}^{2}-\frac{1}{2}(\gamma-1)} \frac{M_{2}^{\prime}}{\bar{M}_{2}} .
\end{array}
$$

However, it is also known that

$$
\frac{p_{3, \mathrm{sh}}}{\bar{p}_{3}}=1+\frac{p_{3}^{\prime}}{\bar{p}_{3}}+\frac{x_{s}^{\prime}}{\bar{p}_{3}} \frac{\mathrm{d} \bar{p}_{3}}{\mathrm{~d} x},
$$

where the relation (B.3) may be substituted into the last term of the equation above. As the conservation of mass flux through a nozzle dictates that

$$
\frac{\mathrm{d} \bar{M}_{3}}{\mathrm{~d} x}=\frac{\bar{M}_{3}\left[1+\frac{1}{2}(\gamma-1) \bar{M}_{3}^{2}\right]}{\bar{M}_{2}\left[1+\frac{1}{2}(\gamma-1) \bar{M}_{2}^{2}\right]}\left(\frac{\bar{M}_{2}^{2}-1}{\bar{M}_{3}^{2}-1}\right)
$$

equation (B.5) may be rewritten as

$$
\frac{p_{3, \mathrm{sh}}}{\bar{p}_{3}}=1+\frac{p_{3}^{\prime}}{\bar{p}_{3}}-x_{s}^{\prime} \gamma \bar{M}_{3}^{2}\left(\frac{\bar{M}_{2}^{2}-1}{\bar{M}_{3}^{2}-1}\right) \frac{1}{\bar{M}_{2}\left[1+\frac{1}{2}(\gamma-1) \bar{M}_{2}^{2}\right]} \frac{\mathrm{d} \bar{M}_{2}}{\mathrm{~d} x} .
$$

Finally, the relationships (B.4) and (B.7) may be compared, resulting in

$$
\frac{p_{3}^{\prime}}{\gamma \bar{p}_{3}}=\frac{x_{s}^{\prime}}{\bar{M}_{2}\left[1+\frac{1}{2}(\gamma-1) \bar{M}_{2}^{2}\right]} F_{p}+\frac{p_{2}^{\prime}}{\gamma \bar{p}_{2}}+\frac{2 \bar{M}_{2}^{2}}{\gamma \bar{M}_{2}^{2}-\frac{1}{2}(\gamma-1)} \frac{M_{2}^{\prime}}{\bar{M}_{2}}
$$

with

$$
\begin{array}{r}
F_{p}=\left[\bar{M}_{2}^{2} \frac{\gamma+3-2 \bar{M}_{2}^{2}}{2 \gamma \bar{M}_{2}^{2}-(\gamma-1)}+\bar{M}_{3}^{2}\left(\frac{\bar{M}_{2}^{2}-1}{\bar{M}_{3}^{2}-1}\right)\right] \frac{\mathrm{d} \bar{M}_{2}}{\mathrm{~d} x} \\
-\frac{2 \bar{M}_{2}^{2}\left[1+\frac{1}{2}(\gamma-1) \bar{M}_{2}^{2}\right]}{\gamma \bar{M}_{2}^{2}-\frac{1}{2}(\gamma-1)} \frac{\mathrm{i} \omega}{\bar{c}_{2}}
\end{array}
$$


Likewise, the velocity and density perturbations just after a shock are

$$
\begin{aligned}
\frac{u_{3}^{\prime}}{\bar{u}_{3}} & =-\frac{x_{s}^{\prime}}{\bar{M}_{2}\left[1+\frac{1}{2}(\gamma-1) \bar{M}_{2}^{2}\right]} F_{u}+\frac{u_{2}^{\prime}}{\bar{u}_{2}}-\frac{2}{1+\frac{1}{2}(\gamma-1) \bar{M}_{2}^{2}} \frac{M_{2}^{\prime}}{\bar{M}_{2}}, \\
\frac{\rho_{3}^{\prime}}{\bar{\rho}_{3}} & =\frac{x_{s}^{\prime}}{\bar{M}_{2}\left[1+\frac{1}{2}(\gamma-1) \bar{M}_{2}^{2}\right]} F_{\rho}+\frac{\rho_{2}^{\prime}}{\bar{\rho}_{2}}+\frac{2}{1+\frac{1}{2}(\gamma-1) \bar{M}_{2}^{2}} \frac{M_{2}^{\prime}}{\bar{M}_{2}}
\end{aligned}
$$

where

$$
\begin{aligned}
& F_{u}=\left(1+\frac{\bar{M}_{2}^{2}-1}{\bar{M}_{3}^{2}-1}\right) \frac{\mathrm{d} \bar{M}_{2}}{\mathrm{~d} x}-\left(1+\bar{M}_{2}^{2}\right) \frac{\mathrm{i} \omega}{\bar{c}_{2}}, \\
& F_{\rho}=\left[2-\bar{M}_{2}^{2}+\bar{M}_{3}^{2}\left(\frac{\bar{M}_{2}^{2}-1}{\bar{M}_{3}^{2}-1}\right)\right] \frac{\mathrm{d} \bar{M}_{2}}{\mathrm{~d} x}-2 \frac{\mathrm{i} \omega}{\bar{c}_{2}} .
\end{aligned}
$$

\section{References}

[1] D. Crighton, A. Dowling, J. Ffowcs-Williams, M. Heckl, F. Leppington, Modern Methods in Analytical Acoustics (Chapter 13), Springer-Verlag, 1992.

[2] M. Howe, Contributions to the theory of aerodynamic sound, with application to excess jet noise and the theory of the flute, Journal of Fluid Mechanics 71 (1975) 625-673.

[3] M. Howe, Indirect combustion noise, Journal of Fluid Mechanics 659 (2010) $267-288$.

[4] W. Strahle, On combustion generated noise, Journal of Fluid Mechanics 49 (1971) 399-414.

[5] N. Cumpsty, Jet engine combustion noise: pressure, entropy and vorticity perturbations produced by unsteady combustion or heat addition, Journal of Sound and Vibration 66 (1979) 527-544.

[6] J. Rayleigh, The theory of sound: Volume II, Dover Publications, 1896.

[7] C. Morfey, Amplification of aerodynamic noise by convected flow inhomogeneities, Journal of Sound and Vibration 31 (1973) 391-397.

[8] J. Ffowcs-Williams, M. Howe, The generation of sound by density inhomogeneities in low mach number nozzle flows, Journal of Fluid Mechanics 70 (1975) 605-622.

[9] M. Howe, The generation of sound by aerodynamic sources in an inhomogeneous steady flow, Journal of Fluid Mechanics 67 (1975) 597-610.

[10] M. Bohn, Response of a subsonic nozzle to acoustic and entropy disturbances, Journal of Sound and Vibration 52 (1977) 283-297. 
[11] F. Marble, S. Candel, Acoustic disturbance from gas non-uniformities convected through a nozzle, Journal of Sound and Vibration 55 (1977) 225-243.

[12] S. Stow, A. Dowling, T. Hynes, Reflection of circumferential modes in a choked nozzle, Journal of Fluid Mechanics 467 (2002) 215-239.

[13] W. Moase, M. Brear, C. Manzie, The forced response of choked nozzles and supersonic diffusers, Journal of Fluid Mechanics 585 (2007) 281-304.

[14] F. Culick, T. Rogers, The response of normal shocks in diffusers, AIAA Journal 21 (1983) 1382-1390.

[15] C.-Y. Kuo, A. Dowling, Oscillations of a moderately underexpanded choked jet impinging upon a flat plate, Journal of Fluid Mechanics 315 (1996) 267291.

[16] N. Cumpsty, F. Marble, The interaction of entropy fluctuations with turbine blade rows; a mechanism of turbojet engine noise, Proceedings of the Royal Society A357 (1977) 323-344.

[17] N. Cumpsty, F. Marble, Core noise from gas turbine exhausts, Journal of Sound and Vibration 54 (1977) 297-309.

[18] T. Sattelmayer, W. Polifke, Assessment of methods for the computation of the linear stability of combustors, Combustion Science and Technology 175 (2003) 453-476.

[19] T. Foglizzo, M. Tagger, Entropic-acoustic instability in shocked accretion flows, Astronomy and Astrophysics 363 (2000) 174-183.

[20] A. Bloy, The pressure waves produced by the convection of temperature disturbances in high subsonic nozzle flows, Journal of Fluid Mechanics 94 (1979) 465-475.

[21] D. Bodony, Scattering of an entropy disturbance into sound by a symmetric thin body, Physics of Fluids 21 (2009) 096101.

[22] J. Miles, Time delay analysis of turbofan engine direct and indirect combustion noise sources, Journal of Propulsion and Power 25 (2009) 218-227.

[23] J. Eckstein, E. Freitag, C. Hirsch, T. Sattelmayer, Experimental study on the role of entropy waves in low-frequency oscillations for a diffusion burner, Proceedings of ASME Turbo Expo (2004) GT2004-54163.

[24] T. Sattelmayer, Influence of the combustor aerodynamics on combustion instabilities from equivalence ratio fluctuations, Journal of Engineering for Gas Turbines and Power 125 (2003) 11-19.

[25] C. Tam, N. Pastouchenko, J. Mendoza, D. Brown, Combustion noise of auxiliary power units, in: 11th AIAA/CEAS Aeroacoutics Conference, AIAA 2005-2829. 
[26] A. Dowling, S. Hubbard, Instability in lean premixed combustors, Proceedings of the IMechE 214 (2000) 317-332.

[27] M. Muthukrishnan, W. Strahle, D. Neale, Separation of hydrodynamic, entropy, and combustion noise in a gas turbine combustor, AIAA Journal 16 (1978) 320-327.

[28] F. Bake, C. Richter, B. Mühlbauer, N. Kings, I. Röhle, F. Thiele, B. Noll, The entropy wave generator (EWG): A reference case on entropy noise, Journal of Sound and Vibration 326 (2009) 574-598.

[29] F. Bake, N. Kings, I. Roehle, Fundamental mechanism of entropy noise in aero-engines: Experimental investigation, Journal of Engineering for Gas Turbines and Power 130 (2008) 011202.

[30] F. Bake, U. Michel, I. Roehle, Investigation of entropy noise in aero-engine combustors, Transactions of the ASME (2007) 370-376.

[31] A. Dowling, S. Stow, Acoustic analysis of gas turbine combustors, Journal of Propulsion and Power 19 (2003) 751-764.

[32] J. Denton, The effects of lean and sweep on transonic fan performance, Task Quarterly (2002) 1-17.

[33] H. Tsien, The transfer function of rocket nozzles, Journal of the American Rocket Society 22 (1952) 139-143. 